\title{
Ontogenetic and spatio-temporal patterns of induced volatiles in Glycine max in the light of the optimal defence hypothesis
}

\author{
Michael Rostás ${ }^{1}$ and Katharina Eggert
}

University of Würzburg, Julius-von-Sachs Institute for Biosciences, Department of Botany II, Juliusvon-Sachs-Platz 3, 97082 Würzburg, Germany

\author{
Postprint version. Original publication in Chemoecology (2008) 18:29-38 \\ ${ }^{1}$ To whom correspondence should be addressed: Tel.: +49 +931 8886223, Fax: +49 +931 888-6235, \\ E-mail: rostas@botanik.uni-wuerzburg.de
}

Summary. Plants attacked by herbivorous insects emit a blend of volatile compounds that serve as important host location cues for parasitoid wasps. Variability in the released blend may exist on the whole-plant and within-plant level and can affect the foraging efficiency of parasitoids. We comprehensively assessed the kinetics of herbivore-induced volatiles in soybean in the context of growth stage, plant organ, leaf age, and direction of signal transport. The observed patterns were used to test the predictions of the optimal defence hypothesis (OD). We found that plants in the vegetative stage emitted 10-fold more volatiles per biomass than reproductive plants and young leaves emitted $>2.6$ times more volatiles than old leaves. Systemic induction in single leaves was stronger and faster by one day in acropetal than in basipetal direction while no systemic induction was found in pods. Herbivore-damaged leaves had a 200 -fold higher release rate than pods. To some extent these findings support the OD: i) indirect defence levels were increased in response to herbivory and ii) young leaves, which are more valuable, emitted more volatiles. However, the fact that reproductive structures emitted no constitutive or very few inducible volatiles is in seeming contrast to the OD predictions. We argue that in case of volatile emission the OD can only partially explain the patterns of defence allocation due to the peculiarity that volatiles act as signals not as toxins or repellents. 
Key words. Indirect plant defence - soybean - Spodoptera frugiperda - tritrophic interactions variability

\section{Introduction}

Plants release volatile secondary metabolites into the environment as a response to feeding damage or oviposition by herbivores (Hilker et al. 2002; Dicke et al. 2003; Rasmann et al. 2005). Volatile emission can be induced by the specific spatio-temporal pattern of damage inflicted by an herbivore and may be enhanced by chemical elicitors present in the herbivore's regurgitate or oviduct secretion (Hilker \& Meiners 2002; Mithöfer et al. 2005; Tumlinson and Lait 2005). Natural enemies such as parasitoids or predators exploit the emitted volatiles as signals to locate their host (Dicke \& Sabelis 1988; Turlings et al. 1990). Since plants can benefit from inducing attractive volatiles, the response is regarded as an indirect defence mechanism (Vet \& Dicke 1992; Kessler and Baldwin 2001). The metabolic costs of producing volatiles in response to herbivore attack are largely outweighed if natural enemies are present (Hoballah et al. 2004). However, substantial ecological costs may incur if volatiles also attract more herbivores (Heil 2004).

The volatile blend released by the attacked plant may vary considerably depending on different abiotic and biotic factors such as nutrient availability, plant genotype or pathogen infection (Gouinguené \& Turlings 2002; Schmelz et al. 2003; Degen et al. 2004; Rostás et al. 2006). So far, only few studies exist that assessed the stability of this plant-parasitoid mutualism with respect to variability. Elevated atmospheric $\mathrm{CO}_{2}$ concentration, for example, was found to reduce the emission of the terpenes (E)-4,8-dimethyl-1,3,7nonatriene and $(E, E)-\alpha$-farnesene. This correlated with an impaired host-searching efficiency of the parasitoid wasp Cotesia plutellae (Vuorinen et al. 2004). Several studies showed that the induced volatile blend of a plant changes with the availability of light. The emission follows a diurnal cycle with lower release rates during the night than during the day (Gouinguené \& Turlings 2002; Loughrin et al. 1994). Also, the growth stage of a plant may determine the quality and quantity of the induced odour bouquet (Gouinguené \& Turlings 2002; Köllner et al. 2004). Zhu \& Park (2005) showed that soybean plants in the early vegetative growth stages V1 and V2 differ slightly in the amounts of released D-limonene and $(E, E)-\alpha$ farnesene.

In addition to differences between individual plants due to biotic and abiotic factors, there is 
within-plant variation in herbivore-induced odour blends, e.g. because volatiles are often emitted not only at the site of damage but also systemically from distant undamaged leaves (Turlings \& Tumlinson 1992). Such odours can differ in composition and intensity from the locally induced blend.

It's a moot question why plants emit spatially heterogenic blends from different tissues instead of synthesizing the maximum amount of the same volatiles by all tissues, which could make the plant more apparent to beneficial parasitoids. This seeming conflict may be resolved if the spatiotemporal pattern of volatile emission follows the predictions of the optimal defence hypothesis (OD). The assumption of the OD is that three main factors: cost of defence, risk of attack and value of the plant organ determine the allocation of defensive secondary metabolites (Rhoades 1979; Stamp 2003). The higher the risk of a given plant tissue to be consumed by herbivores and the higher its value for the plant's fitness, the more energy should be allocated for its defence. Usually, vegetative organs such as leaves are less important for plant fitness than reproductive parts like flowers and fruits, which are also more likely to be attacked (Zangerl \& Bazzaz 1992). Young leaves make a larger contribution to plant fitness than old leaves as they have a higher potential photosynthetic value resulting from a longer expected life-time. In addition, younger leaves are often more nutritious to herbivores (Calvo \& Molina 2005) and should be better defended (Anderson and Agrell 2005).

So far, the predictions of the OD have been developed for and tested on direct rather than indirect defences (Zangerl \& Bazzaz 1992; Zangerl \& Rutledge 1996). The only exceptions are studies that investigated the allocation patterns of extrafloral nectar secretion (Heil et al. 2004; Wäckers \& Bonifay 2004). Hence, the aim of this study was to test the OD assumptions with respect to volatile-mediated indirect defence. Specifically we made the following predictions:

1) The levels of indirect defence are increased in response to herbivory.

2) More valuable plant organs receive a higher defence investment:

a. Young leaves show higher levels of defence than old leaves.

b. Reproductive tissue (fruits) shows higher levels of defence than vegetative tissue (leaves)

To test these predictions, we comprehensively measured the volatile emission patterns of Glycine $\max ($ L.) Merr. (Fabaceae) in response to feeding by caterpillars of Spodoptera frugiperda Smith (Lepidoptera: Noctuidae). We looked at variation on the level of the individual and at within-plant differences. The role of the plant growth stage was assessed by comparing volatile emission of soybeans in the vegetative stage with individuals in the reproductive stage bearing pods, which has not 
been done before. Since $S$. frugiperda may not only feed on leaves but also on pods (Arnett et al. 1981), we asked whether herbivory on leaves or pods, respectively, leads to local and/or systemic release of volatiles in pods. To determine the role of leaf age on volatile emission and additionally to assess the direction and timing of systemic induction volatiles from single leaves were measured.

\section{Materials and methods}

\section{Plants and Insects}

Soybeans, Glycine max var. London, were obtained from Saatbau Linz (Leonding, Austria) and were grown from seed in 9-cm-diameter pots. Plants grew in standard soil containing controlled-release fertilizer (ED 73) in a growth chamber at a 13/11 h photoperiod (300 to $400 \mu \mathrm{mol}$ photons $\mathrm{m}^{-2} \mathrm{~s}^{-1}$ ) with day/night temperature of $28 / 20^{\circ} \mathrm{C}$ and relative humidity of ca. $70 \%$. After eight weeks, plants were fertilized with $50 \mathrm{ml} /$ plant 'Hakaphos blau' (N-P$\mathrm{K}-\mathrm{Mg}=15-10-15-2$; Compo GmbH, Germany) on a weekly base.

Eggs of the fall armyworm, Spodoptera frugiperda, were obtained from Bayer CropScience AG (Monheim, Germany). The hatched larvae were reared in plastic boxes $(20 \times 20 \times 6.5 \mathrm{~cm})$ on artificial diet based on kidney beans (modified from King \& Leppla 1984). Insects were kept in a growth chamber at a $15 / 9 \mathrm{~h}$ photoperiod with day/night temperature of $28 / 24^{\circ} \mathrm{C}$ and relative humidity of ca. $75 \%$. Larvae used in the experiments were five days old (second instar).

\section{Volatile Sampling}

Two different push-pull collection systems were used to perform dynamic headspace sampling of plant volatiles. For collecting volatiles from whole potted soybean plants, a six-arm-olfactometer was used as described by Turlings et al. (2004). Single plants were placed into one of the six glass vessels (volume: $520 \mathrm{ml}$ ) of the olfactometer. Trapping filters were attached to each vessel consisting of glass tubes $(7 \mathrm{~cm})$ containing $30 \mathrm{mg}$ of $80-100$ mesh Super Q adsorbent (Alltech, Deerfield, Illinois, U.S.A.) that was kept in place by two fine mesh metal screens (described by Heath \& Manukian 1992). Filtered (activated charcoal filter, 400 cc, Alltech, Deerfield, Illinois, U.S.A.) and humidified air was pushed into each vessel at a rate of $1.21 \mathrm{~min}^{-1}$ originating from a central in-house compressor. With a vacuum pump (ME2, Vacuubrand, Wertheim, Germany) $0.81 \mathrm{~min}^{-1}$ of air was pulled through the trapping filter. Before each experiment, the traps were cleaned by rinsing with $1 \mathrm{ml}$ methylene chloride. Each collection lasted $3 \mathrm{~h}$ after which the traps were removed, extracted, and analysed.

For collecting volatiles from single leaves in situ, the above described push-pull system was modified. A single leaf of an intact plant was 
inserted into a glass vessel (volume: $320 \mathrm{ml}$ ) that was open on one end. The vessel was held horizontally by a metal support. The petiole of the leaf was wrapped with PTFE-tape. PET-foil (Toppits, Minden, Germany) that was attached around the vessel opening and the wrapped petiole enclosed the leaf airtight in the volatile collection vessel. Clean air was pushed into the vessel while air containing volatiles was pulled through the trapping filters as described above.

Soybean pods grow closely to the stem and thus did not fit into the volatile collection device used for single leaves. Therefore, volatiles emitted by soybean pods were sampled by solid phase microextraction (SPME). Single pods were enclosed in situ in small PET bags $(22 \mathrm{ml})$ with or without caterpillars. The SPME fibre (Supelco Inc., Bellefonte, USA) coated with polydimethylsiloxane $(100 \mu \mathrm{m})$, was activated by inserting it into the GC injector port at $250^{\circ} \mathrm{C}$ for $1 \mathrm{~h}$. Following equilibration, the fibre was introduced into the bag by a small hole that was cut immediately before sampling. Volatiles in the headspace of soybean pods were allowed to adsorb for $1 \mathrm{~h}$. The fibre was then retracted and volatiles were analysed immediately. For control of contaminations, the air in closed empty bags was sampled.

\section{Analysis of Volatiles}

Dynamic headspace sampling: The volatile traps were eluted with $150 \mu \mathrm{l}$ methylene chloride after each collection and two internal standards (n-octane and nonyl acetate, each $200 \mathrm{ng}$ in $10 \mu \mathrm{l}$ methylene chloride) were added to these samples. Aliquots (3 $\mu 1)$ of the samples were analysed by gas chromatography/mass spectrometry (GC: HP 6890N, MSD: Agilent 5975) equipped with a split/splitless injector and a HP-1ms column (30 m x $\quad 0.25 \mathrm{~mm}$ internal diameter, $0.25 \mu \mathrm{m}$ film thickness). Samples were injected in pulsed splitless mode. Inlet temperature was $230^{\circ} \mathrm{C}$. The oven was held at $35^{\circ} \mathrm{C}$ for $3 \mathrm{~min}$ and then programmed at $8^{\circ} \mathrm{C} \min ^{-1}$ to $230^{\circ} \mathrm{C}$, where it was

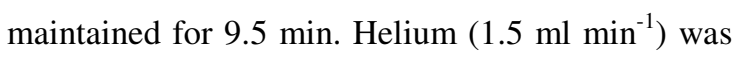
used as carrier gas. Compound identities were confirmed by comparison with mass spectra and retention indices of the Wiley 275 and Massfinder 3/Terpenoids libraries as well as co-injection of standards (Sigma-Aldrich, Germany and Treatt, Suffolk, UK). Identification of $\alpha$-bergamotene was based on library comparisons only. Quantification of compounds was based on comparison with the internal standards. Plants were weighed after the experiment and emission was calculated as $\mathrm{ng}^{-1}$ $\mathrm{FW} \mathrm{h}^{-1}$.

Static headspace sampling: After sampling terminated the SPME fibre was introduced into the injector port of the GC-MS and left there to desorb for $5 \mathrm{~min}$ at $230^{\circ} \mathrm{C}$. Chromatographic analyses were carried out using the GC-MS parameters described above. Volatile quantities were estimated by means of external standard calibration with different 
aliquots $(0.1,0.5,1,5$, and $10 \mathrm{ng})$ of n-octane and nonyl acetate.

\section{Volatile Emissions from Vegetative and Reproductive Growth Stages}

Herbivore-induced volatiles from whole plants were sampled from five-week-old $(\mathrm{V} 3=$ third trifoliate leaf) and ten-week-old (R4 = full pod, 2 $\mathrm{cm}$ pod in top 4 nodes) soybeans. The experiment was started at 10 a.m. by placing 15 larvae $\left(\mathrm{L}_{2}\right)$ of S. frugiperda on each plant. Volatiles were then trapped each day from 10 a.m.-1 p.m. (period 'A') and from 1.30-4.30 p.m. (period 'B'). The last collection was performed $72-75 \mathrm{~h}$ after start. In addition, nocturnally emitted volatiles were collected from ten-week-old plants at 2-5 a.m. (period ' $\mathrm{N}$ ') during three successive nights. The same procedure was carried out with undamaged plants to assess for constitutively emitted volatiles. To check for impurities, empty glass vessels were sampled as blanks. As the extent of feeding damage is positively correlated to volatile synthesis, it was assessed whether caterpillars had fed more on leaves of V3 or R4 plants, respectively. Calculations of consumed leaf areas were carried out by leaf image analysis as described in Rostás et al. (2006).

\section{Volatile Induction in Pods}

Induction of volatiles from soybean pods was performed by placing three larvae of $S$. frugiperda on a pod ( $3^{\text {rd }}$ node), bagged in PET-foil, and left to feed for $20 \mathrm{~h}$. Volatiles were sampled for $1 \mathrm{~h}$ by SPME and then analysed by GC-MS (see above). Systemic induction of pod volatiles was assessed by allowing ten larvae to feed on the first and second leaf, respectively, of a ten-week-old soybean plant. Larvae were confined to the leaves by small PETbags for $72 \mathrm{~h}$ and started feeding at 10 a.m. Sampling of systemically induced pod volatiles was performed at approx. 2 p.m. on the first, second, and third day of feeding. To assess constitutively released volatiles, the headspace of bagged, undamaged soybean pods was sampled following an equilibration time of $20 \mathrm{~h}$. To allow for comparisons, additional SPME measurements were carried out with herbivore-damaged leaves (R4, third trifoliate leaf) in the same manner as described for pods $(n=6)$. Consumption of pod tissue was estimated by measuring the dry weight of caterpillar faeces since image analysis of pods could not be performed due to their threedimensional structure. Faeces were collected from three caterpillars that were placed in a Petri dish with either one pod or one leaf and left to feed for $24 \mathrm{~h}$. The faeces was left to dry for one day at $60^{\circ} \mathrm{C}$.

\section{Rhodamine B Staining}

The vascular architecture of soybean plants was assessed by dye staining. This was done to ensure that volatiles were collected from those two leaves that were directly connected as the intensity of leaf interconnection may influence the strength of 
systemic induction (Orians 2005). Staining was performed by cutting off the lamina of the first (oldest) trifoliate leaf under water and inserting the petiole into a vial $(1.5 \mathrm{ml})$ containing flower foam soaked in rhodamine $\mathrm{B}\left(2.5 \mathrm{mg} \mathrm{ml}^{-1} \mathrm{H}_{2} \mathrm{O}\right.$.). In a second experiment with a new set of plants $(n=6)$, the second trifoliate leaf was used for initial dye application.

\section{Acropetal and Basipetal Induction of Volatiles}

Acropetal and basipetal induction of systemic volatiles was assessed by collecting volatiles from single leaves as described above. For acropetal induction, ten larvae of $S$. frugiperda were enclosed in the vessel that contained the first trifoliate leaf and were left to feed for the duration of the experiment (75 h). Systemically induced volatiles were collected from the second leaf. For basipetal induction of systemic volatiles, larvae fed on the second leaf and systemic volatiles were collected from the first leaf. Five-week-old plants (V3) were used in all experiments $(n=6)$. To check for differences in feeding behaviour, the leaf consumption of caterpillars on the first and second leaves were compared after the experiment was terminated.

\section{Statistical Analysis}

Data were analyzed with STATISTICA 7.1 (StatSoft, Inc., Tulsa, USA). Repeated Measures ANOVA followed by Duncan post-hoc tests was used for comparing volatile emissions. Grouping factors were growth stage and treatment or leaf age and treatment. Time point of sampling was inserted as a repeated measure factor with seven levels. When necessary, data were square root or $\log (x+1)$ transformed to meet the assumptions for analysis of variance (ANOVA). Student's $t$-test was applied for comparison of leaf or pod consumption by caterpillars. Differences were considered to be statistically significant at $\mathrm{p} \leq 0.05$.

\section{Results}

Volatile Emissions from Vegetative and Reproductive Growth Stages

Undamaged soybeans in the V3 stage emitted low amounts of $(E, E)$ - $\alpha$-farnesene $\left(11.0 \pm 2.43 \mathrm{ng} \mathrm{g}^{-1}\right.$ $\left.\mathrm{FW} \mathrm{h}^{-1}\right)$ but also $(Z)$-3-hexenyl acetate $(5.5 \pm 2.81$ $\left.n g \mathrm{~g}^{-1} \mathrm{FW} \mathrm{h}^{-1}\right)$ and methyl salicylate $(3.5 \pm 0.98 \mathrm{ng}$ $\mathrm{g}^{-1} \mathrm{FW} \mathrm{h}^{-1}$ ) during the whole sampling period of 75 h. Plants in the reproductive stage (R4) emitted trace amounts $\left(<1 \mathrm{ng} \mathrm{g}^{-1} \mathrm{FW} \mathrm{h} \mathrm{h}^{-1}\right)$ of the same compounds

Herbivore-damaged soybean plants did not release significantly more volatiles than undamaged controls during the first two sampling periods (day 1 A and B) ( $p>0.05$, Duncan after ANOVA, Tables 1 and 2). Induction of volatiles above constitutive levels could be observed no earlier than 16-19 h after caterpillars started feeding, i.e. during the night session (R4, day $1 \mathrm{~N}$, Fig. 1). With onset 


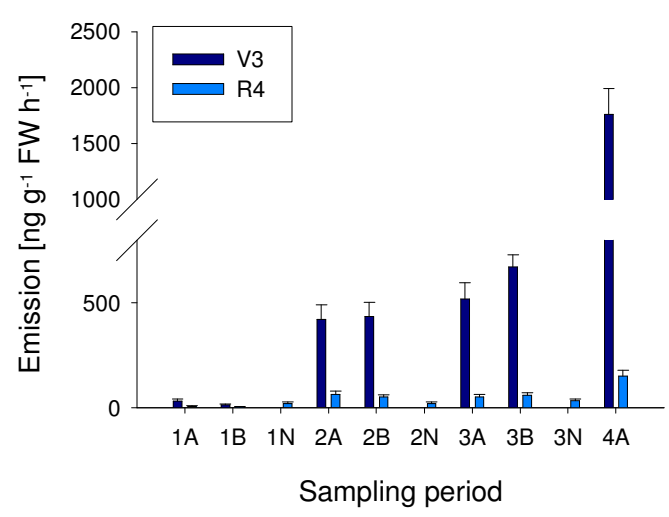

Fig. 1 Total volatile emission from soybean plants over a period of $75 \mathrm{~h}$. Black bars represent total emission from plants in the vegetative stage (V3); grey bars represent total emission from plants in the reproductive stage (R4). The $\mathrm{x}$-axis shows sampling period: number $=$ day, $\mathrm{A}=10$ a.m.-1 p.m., $B=1: 30-4: 30$ p.m., $N=2-5$ a.m. Note that nocturnal volatile blend was measured only from $\mathrm{R} 4$ stage. Means \pm SE are given. For clarity only positive error bars are shown. Repeated Measures ANOVA with Duncan Test; Asterisks indicate significant differences between V3 and R4; *p $\leq 0.05 . \mathrm{n}=6$.

of the light phase on the second day the release rate more than doubled (Fig. 1). Total emission intensified slowly until the afternoon of the third day and increased more than two-fold on day $4 \mathrm{~A}$ (Fig. 1). In principle, this pattern was observed for both growth stages but plants in the V3 stage emitted significantly higher amounts of volatiles than plants in the reproductive stage (Fig. 1, Tables 1 and 2). The differences in emission were significant beginning with day 2 A (Duncan after ANOVA, $p=0.036$ ) and finally at day $4 \mathrm{~A}, \mathrm{~V} 3$ plants emitted $>10$-fold more volatiles per gram biomass than R4 plants (Fig. 1).
During the scotophase, volatile emission was at least $50 \%$ lower than emission during the day (Fig. 1). However, this did not apply to the two sampling periods in the beginning of the experiment when volatiles were not yet induced and therefore at constitutive levels. The same compounds were found during the dark phase as during the photophase but six of the minor compounds $[(Z)-3$ hexenyl iso-butyrate, (Z)-3-hexenyl-2-methyl butanoate, methyl anthranilate, (Z)-jasmone] were below the limit of detection.

The blend composition in the last sampling period consisted of sixteen different volatiles with the sesquiterpene $(E, E)-\alpha$-farnesene $(\mathrm{V} 3=964 \pm$ $103.9 \mathrm{ng} \mathrm{g}^{-1} \mathrm{FW} \mathrm{h}^{-1}[55 \%], \mathrm{R} 4=102 \pm 20.0 \mathrm{ng} \mathrm{g}^{-1}$ FW $\mathrm{h}^{-1}[66 \%]$ ) being the single dominant compound. Other major volatiles, which together represented about one third of the total amount in both stages, were indole, $(Z)$-3-hexenyl acetate, and (Z)-3-hexenyl butyrate. Taking together, seven green leaf volatiles, five sesquiterpenes, three aromatic compounds, and two monoterpenes were found in the headspace of caterpillar-damaged plants. The same blend of volatiles was emitted independently of plant growth stage.

Caterpillars consumed nearly equal amounts of leaf material from both growth stages (V3: $26.6 \pm$ $0.6 \mathrm{~cm}^{2}$ vs. R4: $25.2 \pm 1.3 \mathrm{~cm}^{2}$, Student's $t$-test, $t=$ $0,865, \mathrm{p}>0.05)$ 
Table 1 Effect of growth stage on time-course of herbivore-induced volatile emission.

Values are $\mathrm{ng} \mathrm{g}^{-1} \mathrm{FW} \mathrm{h}^{-1}$. Means $\pm \mathrm{SE}$ are given. V3 = vegetative stage, $\mathrm{R} 4$ = reproductive stage. Total volatile emissions by growth stages were compared using Repeated Measures ANOVA on log $(x+1)$ transformed data (Table 2) followed by Duncan test. Different letters indicate significant differences between V3 and R4 for the same collection period. n.d. = not detected, tr. $=$ traces $(<1 \mathrm{ng}) . \mathrm{n}=6$.

$$
\text { Day } 1 \mathrm{~A}
$$

Compound
Day 1 B

R4
Day 2 A

V3

R4
R4

V3

R4

Fatty acid derivatives

\begin{tabular}{|c|c|c|c|c|c|c|c|c|}
\hline (Z)-3-Hexenal & $3 \pm 0.8$ & $1 \pm 0.1$ & $4 \pm 0.6$ & $1 \pm 0.1$ & $1 \pm 0.2$ & tr. & $1 \pm 0.1$ & tr. \\
\hline (Z)-3-Hexenol & n.d. & n.d. & n.d. & n.d. & $1 \pm 0.8$ & n.d. & tr. & n.d. \\
\hline (Z)-3-Hexenyl acetate & $19 \pm 4.5$ & $1 \pm 0.4$ & $3 \pm 1.1$ & $1 \pm 0.3$ & $24 \pm 4.3$ & $4 \pm 1.0$ & $36 \pm 4.8$ & $3 \pm 0.8$ \\
\hline (Z)-3-Hexenyl propionate & n.d. & n.d. & n.d. & tr. & $5 \pm 0.9$ & tr. & $4 \pm 0.8$ & tr. \\
\hline (Z)-3-Hexenyl isobutyrate & n.d. & n.d. & n.d. & tr. & $1 \pm 0.2$ & tr. & $1 \pm 0.2$ & tr. \\
\hline \multirow{2}{*}{$\begin{array}{l}\text { (Z)-3-Hexenyl butyrate } \\
\text { (Z)-3-Hexenyl } \\
\text { 2-methylbutyrate }\end{array}$} & n.d. & n.d. & n.d. & n.d. & $28 \pm 7.4$ & 8 & $36 \pm 5.7$ & $4 \pm 1.0$ \\
\hline & n.d. & n.d. & n.d. & n.d. & $4 \pm 0.9$ & n.d. & $3 \pm 0.6$ & n.d. \\
\hline \multicolumn{9}{|l|}{ Benzenoids } \\
\hline Indole & n.d. & $2 \pm 1.2$ & $1 \pm 0.4$ & $1 \pm 0.5$ & $107 \pm 19.7$ & $15 \pm 4.0$ & $90 \pm 16.0$ & $10 \pm 2.3$ \\
\hline Methyl salicylate & $1 \pm 0.2$ & $1 \pm 0.3$ & tr. & tr. & n.d. & n.d. & n.d. & n.d. \\
\hline Methyl anthranilate & n.d. & n.d. & n.d. & n.d. & $2 \pm 0.5$ & n.d. & $2 \pm 0.6$ & n.d. \\
\hline \multicolumn{9}{|l|}{ Monoterpenoids } \\
\hline (Z)-Jasmone & n.d. & n.d. & n.d. & n.d. & $7 \pm 1.3$ & n.d. & $3 \pm 0.8$ & tr. \\
\hline (E)-B-Ocimene & n.d. & tr. & n.d. & n.d. & $28 \pm 3.6$ & $4 \pm 1.0$ & $30 \pm 3.3$ & $4 \pm 0.9$ \\
\hline \multicolumn{9}{|l|}{ Sesquiterpenoids } \\
\hline B-Caryophyllene & n.d. & n.d. & n.d. & tr. & $2 \pm 0.2$ & $1 \pm 0.2$ & $1 \pm 0.1$ & tr. \\
\hline a-Humulene & n.d. & n.d. & n.d. & tr. & $1 \pm 0.1$ & tr. & tr. & tr. \\
\hline Germacrene D & n.d. & n.d. & n.d. & tr. & $1 \pm 0.2$ & tr. & n.d. & tr. \\
\hline$\alpha$-Bergamotene & n.d. & n.d. & n.d. & tr. & $1 \pm 0.3$ & tr. & $1 \pm 0.3$ & tr. \\
\hline$(E, E)$ - $\alpha$-Farnesene & $10 \pm 2.5$ & $1 \pm 0.5$ & $6 \pm 1.1$ & $1 \pm 0.4$ & $188 \pm 29.8$ & $32 \pm 8.0$ & $229 \pm 33.2$ & $30 \pm 6.9$ \\
\hline
\end{tabular}


Table 1 (continued)

Day 3 A

Day 3 B

Day 4 A

$\begin{array}{llllllll}\text { Compound } & \text { V3 } & \text { R4 } & \text { V3 } & \text { R4 } & \text { V3 } & \text { R4 }\end{array}$

Fatty acid derivatives

(Z)-3-Hexenal

(Z)-3-Hexenol

$5 \pm 1.3$

Tr.

$5 \pm 1.1$

$1 \pm 0.1$

$6 \pm 1.8$

tr.

(Z)-3-Hexenyl acetate

$5 \pm 2.4$

n.d.

$7 \pm 3.1$

n.d.

$44 \pm 8.9$

n.d.

(Z)-3-Hexenyl propionate

$60 \pm 9.1$

$4 \pm 1.7$

$71 \pm 13.9$

$7 \pm 1.9$

$161 \pm 18.8$

$12 \pm 3.2$

$3 \pm 0.6 \quad$ Tr. $\quad 4 \pm 1.1$

tr.

$22 \pm 3.6$

$1 \pm 0.1$

(Z)-3-Hexenyl isobutyrate

$1 \pm 0.2$

$2 \pm 0.4$

n.d.

$9 \pm 1.8$

tr.

(Z)-3-Hexenyl butyrate

(Z)-3-Hexenyl

$39 \pm 6.4$

$4 \pm 1.2$

$3 \pm 0.8$

$141 \pm 17.5$

$9 \pm 0.1$

$3 \pm 1.1$

n.d.

$6 \pm 1.8$

n.d.

$23 \pm 6.7$

$1 \pm 0.9$

\section{Benzenoids}

Indole

Methyl salicylate

Methyl anthranilate

$55 \pm 10.0$

$6 \pm 1.6$

$93 \pm 16.4$

$6 \pm 1.6$

$261 \pm 49.8$

$17 \pm 2.5$

n.d.

n.d.

n.d.

n.d.

n.d.

n.d.

$1 \pm 0.2$

$2 \pm 0.5$

n.d.

$11 \pm 3.2$

tr.

Monoterpenoids

(Z)-Jasmone

(E)-B-Ocimene

$3 \pm 0.8$

Tr.

$4 \pm 1.2$

n.d.

$18 \pm 3.2$

$1 \pm 0.2$

$28 \pm 3.2$

$4 \pm 1.0$

$33 \pm 5.3$

$4 \pm 0.6$

$80 \pm 9.6$

8

Sesquiterpenoids

B-Caryophyllene

a-Humulene

$2 \pm 0.3 \quad$ Tr.

$1 \pm 0.1$

$1 \pm 0.1$

Tr.

tr.

tr.

tr.

$4 \pm 0.4$

$2 \pm 0.3$

$1 \pm 0.2$

$1 \pm 0.2$

Tr.

$1 \pm 0.1$

a-Bergamotene

$3 \pm 0.5$

Tr.

$3 \pm 0.6$

tr.

$3 \pm 0.3$

tr.

$309 \pm 41.3$

$33 \pm 9.5$

$394 \pm 56.9$

tr.

$9 \pm 1.2$

$1 \pm 0.1$

tr.

$(E, E)$ - $\alpha$-Farnesene

$39 \pm 7.4$

$964 \pm 103.9$

$102 \pm 20.0$ 
Table 2 Effect of growth stage on volatile emission. Results of Repeated Measures ANOVA with dependent variables 'Growth stage' = V3 and R4 and 'Treatment' = control and induced. 'TIME POINT' = sampling period was the repeated factor in the design. Data were $\log (x+1)$ transformed to meet assumptions of normal distribution and variance homogeneity.

\begin{tabular}{lllll} 
Effect & df & MS & F & $p$ \\
\hline & & & & $<0.001$ \\
Growth stage & 1 & 30.310 & 72.212 & $<0.001$ \\
Treatment & 1 & 63.787 & 151.970 & 0.624 \\
Growth stage x Treatment & 1 & 0.104 & 0.247 & $<0.001$ \\
Error & 20 & 0.420 & & $<6.173$ \\
TIME POINT & 6 & 1.655 & 6.295 & $<0.001$ \\
TIME POINT x Growth stage & 6 & 0.157 & 182.524 & $<0.001$ \\
TIME POINT x Treatment & 6 & 4.564 & 10.943 & \\
TIME POINT x Growth stage x Treatment & 6 & 0.274 & &
\end{tabular}

\section{Volatile Induction in Pods}

No volatiles were detected in the headspace of untreated soybean pods. In contrast, nine volatile compounds were found in caterpillar-damaged pods (Fig. 2). Compared to damaged leaves, pods emitted a blend that showed considerable qualitative and quantitative differences: while most compounds were also emitted by leaves, the terpenes linalool and $\alpha$-copaene were detected exclusively in the headspace of pods. Moreover, total emission in pods was about 200 times lower than in SPME-sampled leaves. Due to different methods (SPME versus dynamic headspace) some differences in the ratios of leaf volatiles were apparent, e.g. (E)- $\beta$-ocimene and $\beta$-caryophyllene were more prominent than indole and (Z)-3-hexenyl acetate in leaves sampled by SPME.
Caterpillar feeding on leaves did not induce a systemic release of pod volatiles with the exception of trace amounts of $\alpha$-copaene. However, the emission of this sesquiterpene did not increase with time and was detected only after the first and second day of feeding in half of all sampled pods. No $\alpha$-copaene was found in any of the pods on the third day of sampling.

Caterpillars excreted $3.8 \pm 1.4 \mathrm{mg}$ DW faeces per pod and $9.4 \pm 1.3 \mathrm{mg} \mathrm{DW}$ faeces per leaf (Student's $t$-test after square root transformation, $t=$ 3.38, $\mathrm{p}<0.001)$

\section{Rhodamine B Staining}

Feeding rhodamine $\mathrm{B}$ to the first trifoliate leaf resulted in moderate dye accumulation in the second leaf within $30 \mathrm{~min}$. No other leaves were 
stained at this time point. Two hours later, leaf 2 displayed heavy dye accumulation while moderate accumulation was also found in the younger third leaf. When rhodamine B was fed to the second leaf, the third leaf showed moderate dye accumulation within 30 min. After two hours, heavy staining was observed in leaf 3, while the lower first leaf showed very moderate accumulation of rhodamine B.
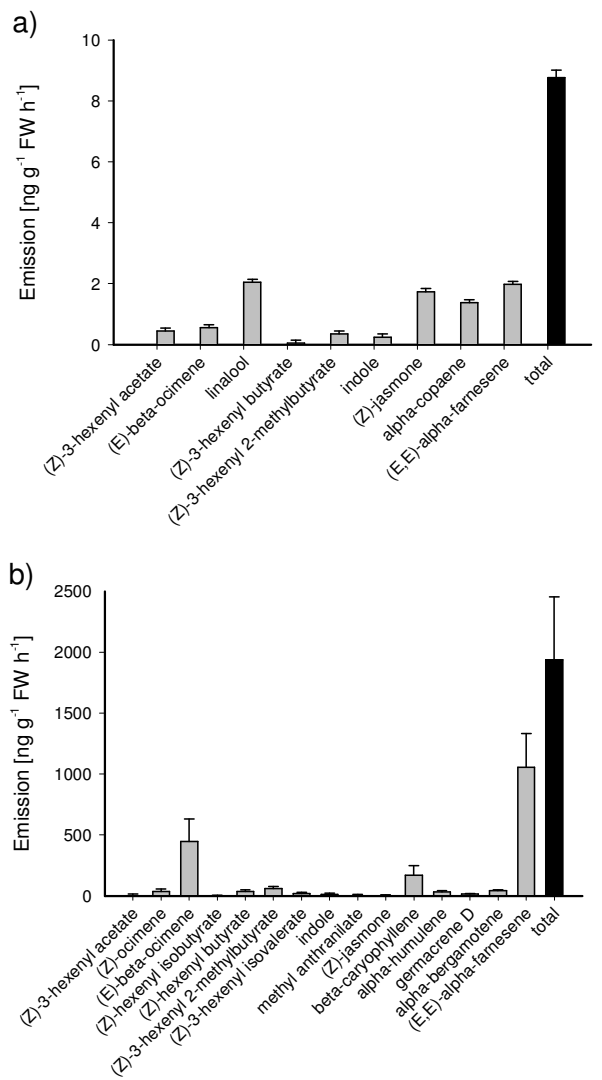

Fig. 2 Herbivore-induced volatiles from soybean pods a) and single leaves b) of an R4-stage plant. Bars represent amounts of volatile compounds emitted by pods after 20 $\mathrm{h}$ of caterpillar feeding. No volatiles were found in undamaged pods from healthy or leaf-damaged plants, respectively. Means $\pm \mathrm{SE}$ are given. For clarity only positive error bars are shown. $\mathrm{n}=6$.

\section{Acropetal and Basipetal Induction of Volatiles}

Acropetal induction. The kinetics of volatile emission from leaf 1 by local induction showed a similar pattern to the emission displayed by whole plants (Fig. 3). Volatiles were observed no earlier than $24 \mathrm{~h}$ after feeding started and increased strongly the next day. Systemically induced volatiles emanating from the orthostichous second trifoliate, appeared one day later (day 3 A) and increased sharply the following day (day 4 A). At this time all afore mentioned compounds were detected except for the green leaf volatiles (Z)-3hexenol and (Z)-3-hexenal. Overall, there were significant leaf age and treatment effects showing that total amounts released from young and locally induced leaves were higher than from old and systemically induced leaves, respectively (ANOVA, leaf age $=p<0.01$, treatment $=p<$ 0.001, Table 3).

Basipetal induction. Feeding damage on the second leaf resulted in a strong emission of volatiles that was more than two-fold higher when compared to the physiologically older first leaf of the same treatment (Fig. 3, Duncan after ANOVA: p $=0.02$ at day 4A). However, basipetal systemic induction of leaf 1 was slower and more attenuated than acropetal induction (Duncan after ANOVA: $p$ $=0.047$ at day $4 \mathrm{~A})$. The main component $(E, E)-\alpha-$ farnesene was always detectable but increased only to a maximum of ca. $50 \mathrm{ng} \mathrm{g}^{-1} \mathrm{FW} \mathrm{h}^{-1}$ at day $3 \mathrm{~A}$. In 
Table 3 Acropetal and basipetal induction of volatiles. Results of Repeated Measures ANOVA with dependent variables 'Leaf age' = leaf 1 and leaf 2 and 'Treatment' = control, locally induced and systemically induced. 'TIME POINT' = sampling period was the repeated factor in the design. Data were $\log (x+1)$ transformed to meet assumptions of normal distribution and variance homogeneity.

$\begin{array}{lllll}\text { Effect } & \text { df } & \text { MS } & \text { F } & p \\ & & & & \\ & & & 5.585 & 0.027 \\ \text { Leaf age } & 1 & 5.510 & 62.089 & <0.001 \\ \text { Treatment } & 2 & 61.254 & 2.426 & 0.110 \\ \text { Leaf age x Treatment } & 2 & 2.393 & 78.739 & <0.001 \\ \text { Error } & 24 & 0.987 & 1.096 & 0.368 \\ \text { TIME POINT } & 6 & 11.721 & 19.380 & <0.001 \\ \text { TIME POINT x Leaf age } & 6 & 0.163 & 4.239 & <0.001 \\ \text { TIME POINT x Treatment } & 12 & 2.885 & & \\ \text { TIME POINT x Leaf x Treatment } & 12 & 0.631 & & \\ \text { Error } & 144 & 0.149 & & \\ \end{array}$
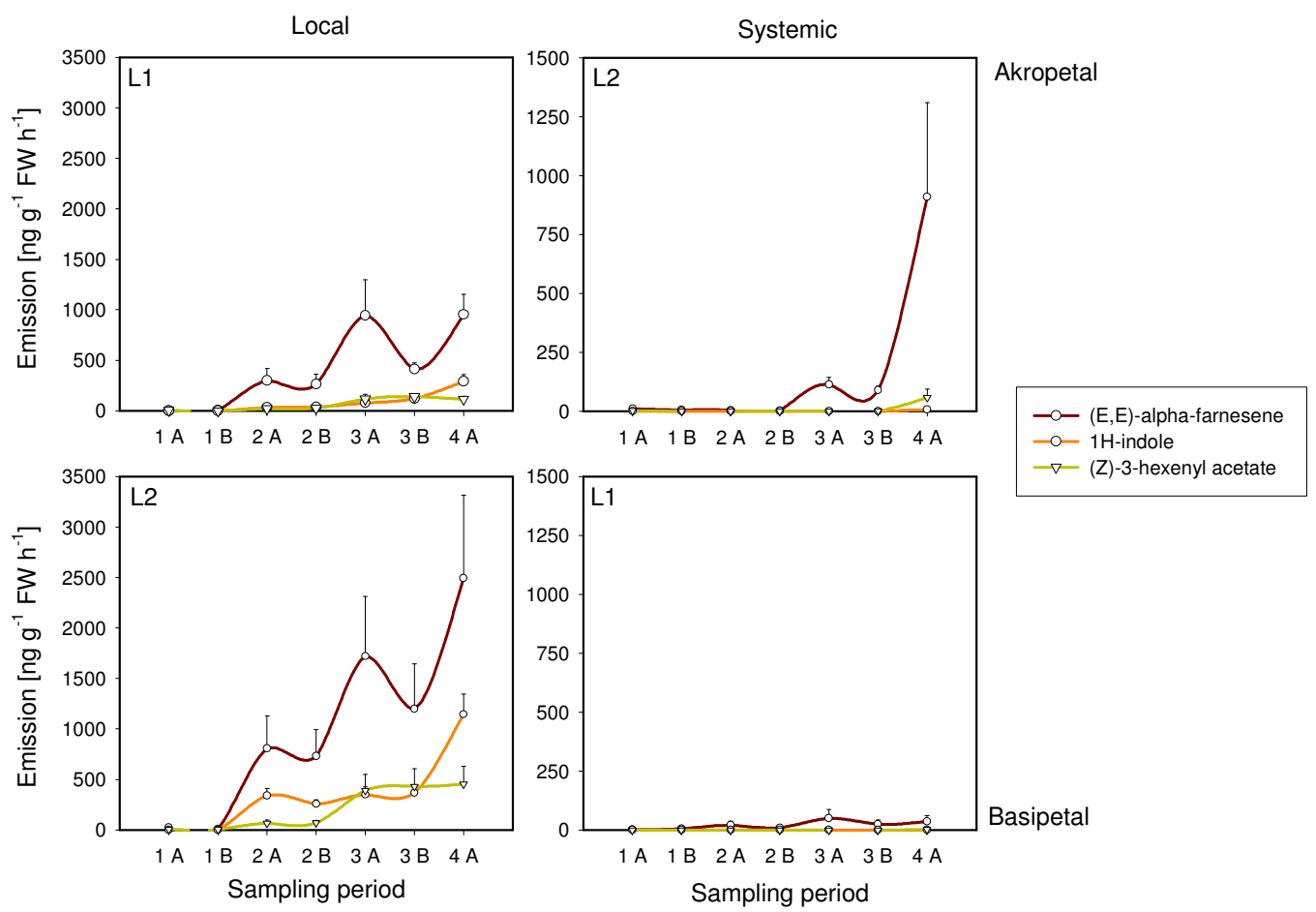

Fig. 3 Kinetics and direction of systemic volatile induction in soybean (V3 stage). Major volatile compounds (ca. 80\% of total blend) from two single leaves. For acropetal induction, locally and systemically induced volatiles were analysed from herbivore-damaged leaf 1 (old), and undamaged leaf 2 (young), respectively. For basipetal induction, the same experiment was carried out vice versa with herbivores feeding on leaf 2 . Means \pm SE are given. For clarity only positive error bars are shown. $\mathrm{n}=6$. 
comparison, the strongest emission of this compound in the systemically induced leaf 2 was 18-fold higher. No other compounds were detected until time point $4 \mathrm{~A}$, at which very low amounts of (Z)-3-hexenyl acetate, (E)- $\beta$-ocimene, and $\beta$ caryophyllene were measured.

The amounts of leaf material consumed by the herbivores on leaf $1\left(20.1 \pm 0.5 \mathrm{~cm}^{2}\right)$ and leaf 2 $\left(19.4 \pm 0.8 \mathrm{~cm}^{2}\right), \quad$ respectively, were not significantly different (Students' $t$ test: $t=0.770, \mathrm{p}$ $>0.05)$

\section{Discussion}

In our study on the indirect defence of G. $\max$ against herbivory, considerable variation in quality and quantity of herbivore-induced volatiles depending on timing, growth stage, plant organ, and leaf age was found. Some of the predictions of the OD were supported but others were not.

Undamaged soybean plants emitted only very low amounts of $(E, E)$ - $\alpha$-farnesene, (Z)-3-hexenyl acetate, and methyl salicylate (MeSA) while continuous feeding caused the release of a characteristic blend of compounds. The induced blend was dominated by the sesquiterpene $(E, E)-\alpha-$ farnesene and the course of emission showed that induced volatiles were not detected until the first night's sampling period. During the next light phase (24 $\mathrm{h}$ after start), a three-fold rise in the amounts of odour compounds was measured from pod-bearing plants (R4-stage) when compared to nocturnal emission. Further increases in the emission rates in both growth stages (V3 and R4) were rather moderate on the third day but more than doubled during the morning of the fourth day. The exponential increase in growth of the leafconsuming caterpillars and subsequently the larger extent of tissue damage was mainly responsible for this rise. It is likely that maximum volatile induction was not yet reached. In those cases where volatiles were measured on the whole plant level (local and systemic emission), the diurnal rhythm was not as evident as in the observed emissions from single leaves, where amounts released between 10 a.m.-1 p.m. were higher than during the period 1:30-4:30 p.m.

Nocturnal release of volatiles was low, with six compounds missing compared to the day blend but emission did not cease completely. Previous studies on maize, cotton, grape vine, Lima bean, peppers and poplar have also shown strong light dependent responses in indirect herbivore defence (Loughrin et al. 1994; Loughrin et al. 1997; Kunert et al. 2002; Gouinguené \& Turlings 2002; Arimura et al. 2004). The de novo synthesis of at least some of the induced terpenes seems to be tightly coupled to photosynthesis as has been demonstrated in labelling studies with $\left[{ }^{13} \mathrm{C}\right] \mathrm{CO}_{2}$ in cotton (Paré \& Tumlinson 1997). An ecological significance for 
nocturnally emitted volatiles was found in tobacco:

De Moraes et al. (2001) demonstrated that some volatiles were released only at night and that this blend repelled ovipositing moths in search for a host plant. Whether or not ecological effects like this can be found in soybean plants, as well, remains to be tested.

Remarkably, the plant's growth stage had a dramatic effect on the strength of volatile emission. Pod-bearing plants emitted ten times less volatiles per biomass than plants in the earlier vegetative stage on the last sampling day. Differences in compound ratios were not found. Such a strong decline in total volatile emission during development has also been described for maize (Köllner et al. 2004). But in contrast to our results, qualitative differences in the emission pattern were found.

No detectable amounts of volatiles were emitted by the pods themselves if plants were left completely unharmed or if caterpillars had fed on leaves (systemic emission). Herbivore-damage on pods led to local emission of volatiles that differed qualitatively and quantitatively from the blend that was released by leaves. For example, two additional terpenes, linalool and $\alpha$-copaene were found. Moreover, damaged pods released ca. $0.5 \%$ of the total amount of damaged leaves when sampled with SPME. This low release rate cannot be explained by caterpillars consuming less tissue from pods than from leaves over the same period of time. In that case, volatile release from pods should have been about $40 \%$ of leaf emission, assuming an approximately linear relationship between extent of damage and volatile emission (Mithöfer et al. 2005). Thus, the release rate was a combination of reduced feeding and a lower inducibility of pods.

Single leaf measurements confirmed that young leaves released higher amounts of volatiles than old leaves and this was irrespective of whether they were induced locally or systemically. In contrast, blend compositions of both leaf ages did not differ. The extent of herbivore damage was ruled out as a reason for variation in volatile emission between young and old leaves because caterpillars had fed equal amounts from both tissue types. Systemic induction of soybean volatiles was highly dependent on the direction of signal transport. Both, acropetal and basipetal induction was observed, but acropetal induction of systemic volatiles was considerably faster and stronger than induction in the opposite direction. In the case of acropetal induction, higher $(E, E)$ - $\alpha$-farnesene levels and the occurrence of several other terpenes was detected $48 \mathrm{~h}$ after caterpillars were allowed to feed on the older first leaf. In contrast, basipetal induction of volatiles was measured not earlier than $72 \mathrm{~h}$ after feeding started. The fact that basipetal induction was found, albeit slower in pace, suggests that the unknown systemic signal is transported via the phloem. This corresponds well with the pattern of phloem transport of rhodamine B into distant 
leaves. Stronger acropetal induction was probably the result of the main direction of flow for assimilates along the prevailing source-sink gradient. Young leaves are often characterised by higher consumption of photosynthates (sink) in contrast to older, fully developed leaves with high assimilation rates (source) (Sitte et al. 1991). Similar results were obtained from gene expression studies in Populus trichocarpa $\mathrm{x}$ deltoides. Arimura et al. (2004) demonstrated strong acropetal induction of a germacrene D synthase while basipetal induction of this gene remained weak and restricted to the neighbouring lower leaves.

How do the described volatile emission patterns fit the OD assumptions? Rhoades (1979) states that if defences are costly, they should be decreased when enemies are absent and increased when plants are attacked (prediction 1). This assumption is clearly supported as no or very low levels of certain volatiles were detected from undamaged organs or entire plants while herbivory induced the synthesis and release of various compounds. In contrast, prediction 2 is only partially corroborated: young leaves, that are more valuable to the plant, also received a higher defence investment than old leaves. This finding was therefore supportive of assumption 2a. However, pods of G. max emitted hardly any volatiles in response to feeding damage. Furthermore, no systemic emission of volatiles from pods was observed and pod-bearing plants released far less compounds than plants in the vegetative stage. This observation is in seeming contradiction to assumption $2 \mathrm{~b}$, which states that the reproductive structures contribute most to a plant's fitness and should be well defended. The latter is the case in Gossypium hirsutum: extrafloral nectar secretion, which attracts predatory arthropods, was constitutive and an order of magnitude higher in bracteal nectaries than in foliar nectaries (Wäckers \& Bonifay 2004). A thinkable reason why volatile emission didn't follow the OD predictions in this aspect may be that volatile emission may be too risky for a plant to rely on when it needs to defend its most valuable organs in terms of fitness. A time lag in protection, which is an inherent property of inducible defences, could be of great disadvantage for offspring production as the herbivore may have already devoured many seeds until it finally gets eliminated by a natural enemy. The OD also implies that reproductive parts receive a higher defence investment by means of constitutive rather than inducible compounds. Again, this does not apply to volatile emission from soybeans as no detectable amounts were released by unharmed pods. In fact, the assumption makes no sense for volatile-mediated induced defences and demonstrates that the OD was originally developed for explaining those defence allocation patterns that act directly on herbivores. A plant emitting large amounts of volatiles in the absence of herbivores is more apparent to its enemies and thus bears higher ecological costs. Furthermore, this 
indirect defence is based on volatiles functioning as a reliable signal indicating the presence of host or prey. In the absence of herbivores, volatiles would mislead foraging enemies and are likely to be ignored in the future since many parasitoids and predators are well capable of associative learning (Degenhardt et al. 2003; Steidle \& van Loon 2003).

More caveats for interpreting volatile patterns in the context of the OD exist. For example, the OD assumes a positive correlation between the amount of defence compounds a plant produces and the attained level of defence. However, we know little about the concentration-dependency of the indirect defence and assuming that more volatiles attract parasitoids faster or in higher numbers may not always be appropriate, although this seems to be the case at least in the well-established maizeSpodoptera spp.-Cotesia marginiventris system (Turlings et al. 2004). Furthermore, it is not clear whether within-plant differences in volatile emission (e.g. local versus systemic emission) are important for natural enemies to locate their prey. It is well possible that volatiles are used exclusively as long-range cues on a whole plant basis and that, once landed on a plant, other strategies are employed to carry out short-range prey location. This is certainly the case in the egg parasitoid Oomyces galerucae, a specialist on the elm leaf beetle (Meiners et al. 2007).

As in other studies that assessed patterns of secondary metabolites in the context of the OD (e.g.
Martin \& Müller 2007) we tested the OD predictions on one particular type of defence. This usual practice has proven successful in several cases. However, one has to bear in mind that if one category of defence fails to follow the OD assumptions in some aspects, as it is the case with herbivore-induced volatiles, this does not mean that a plant will not allocate its resources according to the OD. The hypothesis may possibly be confirmed in all its aspects if the total defence investment of a plant is taken into account instead of focusing on a single group of metabolites. Soybean seeds contain constitutively high concentrations of the isoflavones genistin and daidzin as well as abundant amounts of inducible proteinase inhibitors. These compounds have strong antiherbivore properties (Krishnan 2001; Piubelli et al. 2003). Furthermore, pods bear many trichomes that act against herbivory by small insects (Lam and Pedigo 2001). This shows that the soybean plant does invest a lot into the protection of valuable pods but employs several lines of defence.

In summary, we comprehensively measured herbivore-induced volatile patterns in soybean and tested this data set against the predictions of the OD. To our knowledge this is the first study to specifically apply the OD predictions on this type of indirect defence. Our observations suggest that the OD hypothesis is limited in its ability to explain volatile patterns due to the fact that this category of defence compounds act as inducible signals rather 
than toxins. Their constitutive emission in reproductive structures, as required by the OD, would probably incur only costs but no benefits. Other caveats exist and were also discussed. Further development of plant defence theory will need to take such aspects into account.

\section{Acknowledgements}

The authors thank G. Trautmann (Bayer CropScience AG) for weekly supplies of noctuid eggs. We are very grateful to J. Winkler-Steinbeck for cultivating soybean plants and to O. Frank, who provided technical assistance with GC-MS analyses. We also thank U. Hildebrandt, M. Plessl, C. Müller and J.L.M. Steidle for improving a draft of this manuscript. This research was supported by the Deutsche Forschungsgemeinschaft (Sonderforschungsbereich 567: Mechanismen interspezifischer Interaktionen).

\section{References}

Arnett JD, Bertrand P, Crawford J, Ellis HC, Lambert W, McGlohon N, Suber F, Thompson S, Womack H (1981) Insect and Disease Identification Guide for IPM in the Southeast. Cooperative Extension Service, College of Agriculture: University of Georgia
Anderson P, Agrell J (2005) Within-plant variation in induced defence in developing leaves of cotton plants. Oecologia 144: 1-8

Arimura G, Huber DPW, Bohlmann J (2004) Forest tent caterpillars (Malacosoma disstria) induce local and systemic diurnal emissions of terpenoid volatiles in hybrid poplar (Populus trichocarpa $x$ deltoides): cDNA cloning, functional characterization, and patterns of gene expression of (-)-germacrene D synthase, PtdTPS1. Plant J 37: 603-616

Calvo D, Molina JM (2005) Effects of tangerine (Citrus reticulata) foliage age on Streblote panda larval development and performance. Phytoparasitica 33: 450-459

De Moraes CM, Mescher MC, Tumlinson JH (2001) Caterpillar-induced nocturnal plant volatiles repel conspecific females. Nature 410: $577-580$

Degen T, Dillmann C, Marion-Poll F, Turlings TCJ (2004) High genetic variability of herbivoreinduced volatile emission within a broad range of maize inbred lines. Plant Physiol 135: 19281938

Degenhardt J, Gershenzon J, Baldwin IT, Kessler A (2003) Attracting friends to feast on foes: engineering terpene emission to make crop plants more attractive to herbivore enemies. Curr Op Biotech 14: 169-176

Dicke M, van Poecke RMP, de Boer JG (2003) Inducible indirect defence of plants: from 
mechanisms to ecological functions. Basic Appl Ecol 4: 27-42

Dicke M, Sabelis MW (1988) How plants obtain predatory mites as bodyguards. Netherlands Journal of Zoology 38: 148-165

Gouinguené SP, Turlings TCJ (2002) The effects of abiotic factors on induced volatile emissions in corn plants. Plant Physiol 129: 1296-1307

Heath RR, Manukian A (1992) Development and evaluation of systems to collect volatile semiochemicals from insects and plants using a charcoal-infused medium for air purification. J Chem Ecol 18: 1209-1226

Heil M (2004) Direct defense or ecological costs: Responses of herbivorous beetles to volatiles released by wild lima bean (Phaseolus lunatus). J Chem Ecol 30: 1289-1295

Heil M, Feil D, Hilpert A, Linsenmair KE (2004) Spatiotemporal patterns in indirect defence of a South-East Asian ant-plant support the optimal defence hypothesis. J Trop Ecol 20: $573-580$

Hilker M, Meiners T (2002) Induction of plant responses to oviposition and feeding by herbivorous arthropods: a comparison. Entomol Exp Appl 104: 181-192

Hilker M, Meiners T, Rohfritsch O (2002) The plant's response towards insect oviposition. $\mathrm{Pp}$ 205-228 in Hilker M, Meiners T (eds) Chemoecology of Insect Eggs and Egg Deposition. Berlin: Blackwell Science
Hoballah ME, Köllner TG, Degenhardt J, Turlings TCJ (2004) Costs of induced volatile production in maize. Oikos 105: 168-180

Kessler A, Baldwin IT (2001) Defensive function of herbivore-induced plant volatile emissions in nature. Science 291: 2141-2143

King EG, Leppla NC (1984) Advances and Challenges in Insect Rearing. Washington D.C.: Agriculture Research Service, USDA, U.S. Government Printing Office

Köllner TG, Schnee C, Gershenzon J, Degenhardt J (2004) The sesquiterpene hydrocarbons of maize (Zea mays) form five groups with distinct developmental and organ-specific distribution. Phytochemistry 65: 1895-1902

Krishnan HB (2001) Characterization of a soybean Glycine $\max$ (L.) Merr. mutant with reduced levels of Kunitz trypsin inhibitor. Plant Sci 160: $979-986$

Kunert M, Biedermann A, Koch T, Boland W (2002) Ultrafast sampling and analysis of plant volatiles by a hand-held miniaturised GC with pre-concentration unit: Kinetic and quantitative aspects of plant volatile production. J Sep Sci 25:677-684

Lam WKF, Pedigo LP (2001) Effect of trichome density on soybean pod feeding by adult bean leaf beetles (Coleoptera: Chrysomelidae). J Econ Entomol 94: 1459-1463

Loughrin JH, Manukian A, Heath RR, Turlings TCJ, Tumlinson JH (1994) Diurnal cycle of 
emission of induced volatile terpenoids herbivore-injured cotton plants. Proc Natl Acad Sci USA 91: 11836-11840

Loughrin JH, Potter DA, HamiltonKemp TR, Byers ME (1997) Diurnal emission of volatile compounds by Japanese beetle-damaged grape leaves. Phytochemistry 45: 919-923

Meiners T, Büchel K, Kühnle A, Kühn M, Tillmann K, Hilker M (2007) Temporal, spatial and quantitative variation of beetle egg deposition on elms affects attraction of egg parasitoids. Abstract of the oral presentation at the $13^{\text {th }}$ Symposium on Insect-Plant Relationships, Uppsala, Sweden

Martin N, Müller C (2007) Induction of plant responses by a sequestering insect: Relationship of glucosinolate concentration and myrosinase activity. Basic Appl Ecol 8: $13-25$

Mithöfer A, Wanner G, Boland W (2005) Effects of feeding Spodoptera littoralis on lima bean leaves. II. Continuous mechanical wounding resembling insect feeding is sufficient to elicit herbivory-related volatile emission. Plant Physiol 137: 1160-1168

Orians C (2005) Herbivores, vascular pathways, and systemic induction: Facts and artifacts. J Chem Ecol 31: 2231-2242

Paré PW, Tumlinson JH (1997) De novo biosynthesis of volatiles induced by insect herbivory in cotton plants. Plant Physiol 114: $1161-1167$

Piubelli GC, Hoffmann-Campo CB, De Arruda IC, Franchini JC, Lara FM (2003) Flavonoid increase in soybean as a response to Nezara viridula injury and its effect on insect-feeding preference. J Chem Ecol 29: 1223-1233

Rasmann S, Köllner TG, Degenhardt J, Hiltpold I, Toepfer S, Kuhlmann U, Gershenzon J, Turlings TCJ (2005) Recruitment of entomopathogenic nematodes by insectdamaged maize roots. Nature 434: 732-737

Rhoades DF (1979) Evolution of plant chemical defence against herbivores. Pp 3-54 in Rosenthal GA, Janzen DH (eds) Herbivores: Their Interaction with Secondary Plant Metabolites. New York: Academic Press

Rostás M, Ton J, Mauch-Mani B, Turlings TCJ (2006) Fungal infection reduces herbivoreinduced plant volatiles of maize but does not affect naive parasitoids. J Chem Ecol 32: 1897-1909

Schmelz EA, Alborn HT, Engelberth J, Tumlinson JH (2003) Nitrogen deficiency increases volicitin-induced volatile emission, jasmonic acid accumulation, and ethylene sensitivity in maize. Plant Physiol 133: 295-306

Sitte P, Ziegler H, Ehrendorfer F, Bresinsky A (1991) Lehrbuch der Botanik für Hochschulen. Stuttgart: Gustav Fischer Verlag 
Stamp N (2003) Theory of plant defensive level: example of process and pitfalls in development of ecological theory. Oikos 102: 672-678

Steidle JLM, van Loon JJA (2003) Dietary specialization and infochemical use in carnivorous arthropods: testing a concept. Entomol Exp Appl 108: 133-148

Tumlinson JH, Lait CG (2005) Biosynthesis of fatty acid amide elicitors of plant volatiles by insect herbivores. Arch Insect Biochem Physiol 58: $54-68$

Turlings TCJ, Tumlinson JH (1992) Systemic release of chemical signals by herbivoreinjured corn. Proc Natl Acad Sci USA 89: 8399-8402

Turlings TCJ, Tumlinson JH, Lewis WJ (1990) Exploitation of herbivore-induced plant odors by host-seeking parasitic wasps. Science 250 : $1251-1253$

Turlings TCJ, Davison AC, Tamò C (2004) A sixarm olfactometer permitting simultaneous observation of insect attraction and odour trapping. Physiol Entomol 29: 45-55

Vet LEM, Dicke M (1992) Ecology of infochemical use by natural enemies in a tritrophic context. Annu Rev Entomol 37: 141-172

Wäckers FL, Bonifay C (2004) How to be sweet? Extrafloral nectar allocation by Gossypium hirsutum fits optimal defense theory predictions. Ecology 85: 1512-1518

Vuorinen T, Nerg AM, Ibrahim MA, Reddy GVP, Holopainen JK (2004) Emission of Plutella xylostella-induced compounds from cabbages grown at elevated $\mathrm{CO} 2$ and orientation behavior of the natural enemies. Plant Physiol 135: $1984-1992$

Zangerl AR, Bazzaz FA (1992) Theory and pattern in plant defense allocation. Pp 363-391 in Fritz RS, Simms EL (eds) Plant Resistance to Herbivore and Pathogens: Ecology, Evolution, and Genetics. Chicago: University of Chicago Press

Zangerl AR, Rutledge CE (1996) The probability of attack and patterns of constitutive and induced defense: a test of optimal defense theory. Am Nat 147: 599-608

Zhu JW, Park KC (2005) Methyl salicylate, a soybean aphid-induced plant volatile attractive to the predator Coccinella septempunctata. $\mathrm{J}$ Chem Ecol 31: 1733-1746 